\title{
Fixed points of periodic mappings in Hilbert spaces
}

\begin{abstract}
In this paper we give new estimates for the Lipschitz constants of $n$-periodic mappings in Hilbert spaces, in order to assure the existence of fixed points and retractions on the fixed point set.
\end{abstract}

1. Introduction. In order to assure the existence of fixed points for a continuous mapping on Banach spaces, we need to impose some conditions on the mapping or on the Banach space. We will deal with $k$-Lipschitzian mappings:

Definition 1.1. Let $T: C \rightarrow C$ be a mapping with $C$ a nonempty, closed and convex subset of a Banach space $X$. T is called a Lipschitzian mapping if there is $k>0$ such that

$$
\|T x-T y\| \leq k\|x-y\|
$$

holds for any $x, y \in C$ and we will write $T \in \mathscr{L}(k)$. If $k_{0}$ is the smallest number such that $T \in \mathscr{L}(k)$, we will write $T \in \mathscr{L}_{0}\left(k_{0}\right)$.

Definition 1.2. Let $T: C \rightarrow C$ where $C$ is a nonempty, closed and convex subset of a Banach space $X$. If $T^{n}=I, T$ is called an $n$-periodic mapping.

In 1981 K. Goebel and M. Koter, see [1, pp. 179-180], proved the following theorem which shows that the condition of periodicity for nonexpansive mappings is very strong:

2000 Mathematics Subject Classification. 47H10, 47H09.

Key words and phrases. Fixed point, retractions, periodic mappings.

This work was partly supported by CIMAT and by Conacyt scholarship 170778 . 
Theorem 1.3. If $C$ is a nonempty, closed and convex subset of a Banach space, then any nonexpansive n-periodic mapping $T: C \rightarrow C$ has a fixed point.

This covers the case $k \leq 1$, and thus we will study $n$-periodic and $k$ Lipschitzian mappings with $k>1$.

Remark 1.4. If $T$ is $n$-periodic, then $\operatorname{Fix}(T)=\operatorname{Fix}\left(T^{n-1}\right)$. In fact, if $x \in \operatorname{Fix}(T)$, then it is clear that $x \in \operatorname{Fix}\left(T^{n-1}\right)$ and if $x \in \operatorname{Fix}\left(T^{n-1}\right)$, then $T\left(T^{n-1} x\right)=T x$, that is, $x=T x$.

Therefore, we will only consider $n$-periodic mappings $T \in \mathscr{L}_{0}(k)$ such that $T^{n-1} \in \mathscr{L}_{0}(p)$ with $p \geq k$ because if $p<k$, we will work with $T^{n-1}$ instead of $T$.

Let us define the following number:

$$
\gamma_{n}^{X}=\inf \left\{k: \exists(C \subset X, T: C \rightarrow C), T^{n}=I, T \in \mathscr{L}_{0}(k), \operatorname{Fix}(T)=\emptyset\right\},
$$

where $C$ is a nonempty, closed and convex subset of a Banach space $X$. In 1981 K. Goebel and M. Koter [1, pp. 179-180] showed that for any $n$, $\gamma_{n}^{X}>1$.

In $1971 \mathrm{~K}$. Goebel and E. Złotkiewicz [2] proved that if $k<2$, then $\operatorname{Fix}(T) \neq \emptyset$ for 2-periodic and $k$-Lipschitzian mappings in general Banach spaces $X$, that is, $\gamma_{2}^{X} \geq 2$.

Furthermore, in 1986 M. Koter (see also [4]) proved that $\gamma_{2}^{H} \geq \sqrt{\pi^{2}-3}$ $\approx 2.6209$ for Hilbert spaces $H$.

In 2005 J. Górnicki and K. Pupka [3] gave estimations of $\gamma_{n}^{X}$ for $n \geq 3$ for any Banach space $X$, in particular $\gamma_{3}^{X} \geq 1.3821, \gamma_{4}^{X} \geq 1.2524$ and $\gamma_{5}^{X} \geq 1.1777$. These are the best estimations known nowadays for general Banach spaces; we will improve these estimations for Hilbert spaces.

2. Estimations of $\gamma_{n}^{H}$ in Hilbert spaces. The following lemma gives conditions for the existence of fixed points and retractions on the fixed point set:

Lemma 2.1. Let $X$ be a complete metric space and $T: X \rightarrow X$ a continuous mapping. Suppose there are $u: X \rightarrow X, 0<A<1$ and $B>0$, such that for every $x \in X$ :

(i) $\mathrm{d}(T u(x), u(x)) \leq A \mathrm{~d}(T x, x)$,

(ii) $\mathrm{d}(u(x), x) \leq B \mathrm{~d}(T x, x)$.

Then $\operatorname{Fix}(T) \neq \emptyset$.

If we define $R(x)=\lim _{n \rightarrow \infty} u^{n}(x)$ and $u$ is a continuous mapping, then $R$ is a retraction from $X$ to $\operatorname{Fix}(T)$.

If additionally $u \in \mathscr{L}(p)$ :

(a) If $p<1$, then $T$ has a unique fixed point.

(b) If $p=1$, then $R$ is a nonexpansive mapping. 
(c) If $p>1$ and $D=\operatorname{diam}(X)<\infty$, then $R$ is a Hölder continuous retraction from $X$ to $\operatorname{Fix}(T)$.

Proof. Górnicki in [3] proved that if (i) and (ii) hold and $x \in X$, the sequence $\left\{u^{n}(x)\right\}_{n=1}^{\infty}$ converges to a fixed point of $T$. Furthermore, for every $m, n \in \mathbb{N}$,

$$
\mathrm{d}\left(u^{n+m}(x), u^{n}(x)\right) \leq B A^{n} \frac{1}{1-A} \mathrm{~d}(T x, x) .
$$

If $x \in \operatorname{Fix}(T), R x=x$, since clearly $\operatorname{Fix}(u)=\operatorname{Fix}(T)$. Thus, in order to prove that $R$ is a retraction, we only need to show that $R$ is a continuous mapping.

Let $E_{L}=\{x \in X: \mathrm{d}(x, T x)<L\}$. Then $X=\bigcup_{L} E_{L}$. For $x \in E_{L}$, by (2.1) we have

$$
\mathrm{d}\left(R(x), u^{n}(x)\right) \leq \frac{B A^{n}}{1-A} \mathrm{~d}(T x, x)<\frac{L B A^{n}}{1-A} .
$$

Since the last inequality does not depend on $x$, and since $A<1, u^{n}$ converges uniformly to $R$ on $E_{L}$, and hence $R$ is continuous in $X$.

(a) If $p<1$, then $u$ is a contraction and has a unique fixed point, hence $T$ has a unique fixed point.

(b) If $p=1, \mathrm{~d}(R x, R y)=\lim _{n \rightarrow \infty} \mathrm{d}\left(u^{n}(x), u^{n}(y)\right) \leq \mathrm{d}(x, y)$.

(c) Let $p>1$ and $D=\operatorname{diam}(X)$. For any $n \in \mathbb{N}$ and any $x, y \in X$ we have

$$
\begin{aligned}
\mathrm{d}(R x, R y) & \leq \mathrm{d}\left(R x, T^{n} x\right)+\mathrm{d}\left(T^{n} x, T^{n} y\right)+\mathrm{d}\left(T^{n} y, R y\right) \\
& \leq B A^{n} \frac{1}{1-A}(\mathrm{~d}(T x, x)+\mathrm{d}(T y, y))+p^{n} \mathrm{~d}(x, y) \\
& \leq \frac{2 D B}{1-A} A^{n}+p^{n} \mathrm{~d}(x, y)=E(n) .
\end{aligned}
$$

Let us define $n_{0} \in \mathbb{N}$ as follows:

$$
n_{0}=\min \left\{n \in \mathbb{N}: \mathrm{d}(x, y) p^{n} \geq \frac{2 D B A^{n}}{1-A}\right\} .
$$

If $\mathrm{d}(x, y) \geq \frac{2 D B}{1-A}$, then we have $n_{0}=0$, and $\mathrm{d}(R x, R y) \leq 2 \mathrm{~d}(x, y)$. Suppose that we have $\mathrm{d}(x, y)<\frac{2 D B}{1-A}$, then $n_{0}>0$. With

$$
s_{0}=\frac{\ln \left(\frac{2 D B}{(1-A) \mathrm{d}(x, y)}\right)}{\ln (p)+\ln (1 / A)},
$$

we have the equality

$$
\mathrm{d}(x, y) p^{s_{0}}=\frac{2 D B}{1-A} A^{s_{0}},
$$


hence $n_{0}-1<s_{0} \leq n_{0}$ and there is $0 \leq r_{0}<1$ such that $n_{0}=s_{0}+r_{0}$. In consequence we have

$$
n_{0}=\frac{\ln \left(\frac{2 D B}{(1-A) \mathrm{d}(x, y)} e^{(\ln (p)+\ln (1 / A)) r_{0}}\right)}{\ln (p)+\ln (1 / A)}
$$

and

$$
\begin{aligned}
E\left(n_{0}\right) & =\mathrm{d}(x, y) p^{n_{0}}+A^{n_{0}} \frac{2 D B}{1-A} \\
& \leq 2 \mathrm{~d}(x, y) p^{n_{0}} \\
& \leq 2\left(\frac{2 D B}{1-A}\right)^{\frac{\ln (p)}{\ln (p)+\ln (1 / A)}} p \mathrm{~d}(x, y)^{\frac{\ln (1 / A)}{\ln (p)+\ln (1 / A)}} .
\end{aligned}
$$

The following lemma is a generalization of the parallelogram law for Hilbert spaces, and we will use it throughout this paper:

Lemma 2.2. Let $H$ be a Hilbert space and let $n \in \mathbb{N}$ and $a_{i} \in[0,1]$ for $i=1, \ldots, n$, such that $\sum_{i=1}^{n} a_{i}=1$. If $x_{i} \in H$ for $i=1, \ldots, n$, then

$$
\left\|\sum_{i=1}^{n} a_{i} x_{i}\right\|^{2}=\sum_{i=1}^{n} a_{i}\left\|x_{i}\right\|^{2}-\sum_{1 \leq i<j \leq n} a_{i} a_{j}\left\|x_{i}-x_{j}\right\|^{2} .
$$

Proposition 2.3. Let $n \in \mathbb{N}$ and $T: C \rightarrow C$ be n-periodic and $k$-Lipschitzian mapping, where $C$ is a nonempty, closed and convex subset of a Hilbert space. Let $a_{i}>0$ for $i=1, \ldots, n$, such that $\sum_{i=1}^{n} a_{i}=1$. Let us define $a_{0}=a_{n}$. If for $x \in C$ we define

$$
z=\sum_{i=1}^{n} a_{i} T^{i} x
$$

then we have $\|z-x\| \leq \sum_{i=1}^{n-1} a_{i}\left\|T^{i} x-x\right\|$ and

$$
\|z-T z\|^{2} \leq \sum_{0 \leq j<i \leq n-1} F\left(k, a_{j}, a_{j+1}, a_{i}, a_{i+1}\right)\left\|T^{j} x-T^{i} x\right\|^{2},
$$

where $F(k, x, y, u, w)=k^{2}(y u+x w-x u)-x u$. 
Proof. With $a_{i}, x$ and $z$ as above, the first inequality is trivial. Now let $L=\sum_{0 \leq j<i \leq n-1} a_{i} a_{j}\left\|T^{i} x-T^{j} x\right\|^{2}$. By the previous lemma we have

$$
\begin{aligned}
\|z-T z\|^{2} & =\left\|\sum_{i=1}^{n} a_{i}\left(T^{i} x-T z\right)\right\|^{2} \\
& =\sum_{i=1}^{n} a_{i}\left\|T^{i} x-T z\right\|^{2}-\sum_{0 \leq j<i \leq n-1} a_{i} a_{j}\left\|T^{i} x-T^{j} x\right\|^{2} \\
& \leq k^{2} \sum_{s=1}^{n} a_{s}\left\|z-T^{s-1} x\right\|^{2}-L \\
& =k^{2} \sum_{s=1}^{n} a_{s}\left\|\sum_{i=1}^{n} a_{i}\left(T^{i} x-T^{s-1} x\right)\right\|^{2}-L \\
& =k^{2} \sum_{s=1}^{n} a_{s}\left(\sum_{i=1}^{n} a_{i}\left\|T^{i} x-T^{s-1} x\right\|^{2}\right)-\left(k^{2}+1\right) L .
\end{aligned}
$$

The first term of the last expression is equal to

$$
\begin{aligned}
& k^{2} \sum_{s=1}^{n} a_{s}\left(\sum_{i=1}^{n} a_{i}\left\|T^{i} x-T^{s-1} x\right\|^{2}\right)=k^{2} \sum_{s=0}^{n-1} a_{s+1}\left(\sum_{i=1}^{n} a_{i}\left\|T^{i} x-T^{s} x\right\|^{2}\right) \\
& =k^{2} \sum_{s=1}^{n-1} a_{s+1}\left(\sum_{i=1}^{n} a_{i}\left\|T^{i} x-T^{s} x\right\|^{2}\right)+k^{2} a_{1} \sum_{i=1}^{n} a_{i}\left\|T^{i} x-x\right\|^{2} \\
& =k^{2} \sum_{s=1}^{n-1} a_{s+1}\left(\sum_{i=1}^{n-1} a_{i}\left\|T^{i} x-T^{s} x\right\|^{2}\right)+k^{2} a_{1} \sum_{i=1}^{n} a_{i}\left\|T^{i} x-x\right\|^{2} \\
& \quad+k^{2} \sum_{s=1}^{n-1} a_{s+1} a_{n}\left\|x-T^{s} x\right\|^{2} \\
& =k^{2} \sum_{1 \leq s<i \leq n-1}\left(a_{s+1} a_{i}+a_{s} a_{i+1}\right)\left\|T^{i} x-T^{s} x\right\|^{2}+k^{2} a_{1} \sum_{i=1}^{n} a_{i}\left\|T^{i} x-x\right\|^{2} \\
& +k^{2} \sum_{s=1}^{n-1} a_{s+1} a_{n}\left\|x-T^{s} x\right\|^{2} \\
& =k^{2} \sum_{0 \leq s<i \leq n-1}\left(a_{s+1} a_{i}+a_{s} a_{i+1}\right)\left\|T^{i} x-T^{s} x\right\|^{2} .
\end{aligned}
$$


Hence

$$
\begin{aligned}
\|z-T z\|^{2} & \leq \sum_{0 \leq j<i \leq n-1}\left[k^{2}\left(a_{j+1} a_{i}+a_{j} a_{i+1}-a_{j} a_{i}\right)-a_{j} a_{i}\right]\left\|T^{j} x-T^{i} x\right\|^{2} \\
& =\sum_{0 \leq j<i \leq n-1} F\left(k, a_{j}, a_{j+1}, a_{i}, a_{i+1}\right)\left\|T^{j} x-T^{i} x\right\|^{2} .
\end{aligned}
$$

Applying the last result we have the following:

Proposition 2.4. Let $C$ be a nonempty, closed and convex subset of a Hilbert space $H$ and $T: C \rightarrow C, T \in \mathscr{L}(k)$ be an n-periodic mapping, with $n \geq 3$. For $x \in C$ let us define

$$
u=\frac{1}{n}\left(x+T x+\ldots+T^{n-1} x\right),
$$

then $u \in \mathscr{L}\left(\frac{1+k+\ldots+k^{n-1}}{n}\right)$ and

$$
\begin{aligned}
\|u-T u\|^{2} & \leq \frac{1}{n^{2}}\left[\left(k^{2}-1\right) k^{2(n-1)}+\sum_{j=2}^{n-1}\left(k^{2 j}-1\right)\left(\frac{k^{n-j}-1}{k-1}\right)^{2}\right]\|x-T x\|^{2} \\
& =A(k)\|x-T x\|^{2} .
\end{aligned}
$$

Thus, if $A(k)<1$, then $\operatorname{Fix}(T) \neq \emptyset$ and $\operatorname{Fix}(T)$ is a retract of $C$. If $k=1$, $\operatorname{Fix}(T)$ is a nonexpansive retract of $C$ and if $k>1$ with $C$ bounded, $\operatorname{Fix}(T)$ is a Hölder continuous retract of $C$.

Proof. From Proposition 2.3, taking $a_{i}=\frac{1}{n}$ we get

$$
\|u-T u\|^{2} \leq \sum_{0 \leq j<i \leq n-1} \frac{k^{2}-1}{n^{2}}\left\|T^{j} x-T^{i} x\right\|^{2} .
$$

Since for $j<i,\left\|T^{j} x-T^{i} x\right\| \leq k^{i-j}\left\|x-T^{i-j}\right\|$, for $j<n-1$,

$$
\left\|x-T^{j} x\right\| \leq \sum_{i=0}^{j-1}\left\|T^{i} x-T^{i+1} x\right\| \leq \sum_{i=0}^{j-1} k^{i}\|x-T x\|=\frac{k^{j}-1}{k-1}\|x-T x\|
$$

and

$$
\left\|x-T^{n-1} x\right\|=\left\|T^{n} x-T^{n-1} x\right\| \leq k^{n-1}\|T x-x\|,
$$

we get 


$$
\begin{aligned}
\|u-T u\|^{2} & \leq \frac{k^{2}-1}{n^{2}} \sum_{j=0}^{n-2} \sum_{i=j+1}^{n-1}\left\|T^{j} x-T^{i} x\right\|^{2} \\
& \leq \frac{k^{2}-1}{n^{2}} \sum_{j=0}^{n-2} k^{2 j} \sum_{i=1}^{n-j-1}\left\|x-T^{i} x\right\|^{2} \\
& =\frac{k^{2}-1}{n^{2}} \sum_{i=1}^{n-1}\left(\sum_{j=0}^{n-i-1} k^{2 j}\right)\left\|x-T^{i} x\right\|^{2} \\
& =\sum_{i=1}^{n-1} \frac{k^{2(n-i)}-1}{n^{2}}\left\|x-T^{i} x\right\|^{2} .
\end{aligned}
$$

Thus

$$
\begin{aligned}
\| u & -T u\left\|^{2} \leq \frac{1}{n^{2}} \sum_{j=1}^{n-1}\left(k^{2(n-j)}-1\right)\right\| x-T^{j} x \|^{2} \\
& =\frac{1}{n^{2}} \sum_{j=1}^{n-2}\left(k^{2(n-j)}-1\right)\left\|x-T^{j} x\right\|^{2}+\frac{1}{n^{2}}\left(k^{2}-1\right)\left\|x-T^{n-1} x\right\|^{2} \\
& \leq \frac{1}{n^{2}}\left[\left(k^{2}-1\right) k^{2(n-1)}+\sum_{j=2}^{n-1}\left(k^{2 j}-1\right)\left(\frac{k^{n-j}-1}{k-1}\right)^{2}\right]\|x-T x\|^{2} .
\end{aligned}
$$

By Lemma 2.1, if $A(k)<1, \operatorname{Fix}(T) \neq \emptyset$ and is a retract of $C$. Also if $k=1, \operatorname{Fix}(T)$ is a nonexpansive retract of $C$ and if $k>1$ and $C$ is bounded, $\operatorname{Fix}(T)$ is a Hölder continuous retract of $C$.

Since for fixed $n, \lim _{k \rightarrow 1} A(k)=0$, there is $k>1$ such that $A(k)<1$, and this is another proof that for Hilbert spaces $H, \gamma_{n}^{H}>1$.

In 2000, M. Koter [5] gave the following estimations: $\gamma_{3}^{H} \geq 1.3666, \gamma_{4}^{H} \geq$ 1.1962 and $\gamma_{5}^{H} \geq 1.0849$. But her procedure cannot be applied in order to estimate $\gamma_{n}^{H}$ if $n>6$. In 2005 J. Górnicki and K. Pupka [3] gave the following estimations in general Banach spaces: $\gamma_{3}^{X} \geq 1.3821, \gamma_{4}^{X} \geq 1.2524$, $\gamma_{5}^{X} \geq 1.1777$ and $\gamma_{6}^{X} \geq 1.1329$.

Applying (2.5) for $n=3$, if $k$ satisfies the inequality

$$
\frac{1}{9}\left(\left(k^{2}-1\right) k^{4}+\left(k^{4}-1\right)\right)<1,
$$

then we have a fixed point. Thus $\gamma_{3}^{H} \geq 1.4678$; similarly we get $\gamma_{4}^{H} \geq 1.2905$.

For $n \geq 5, j=1$ and $i=n-1$, the estimate in (2.2) improves if we take

$$
\left\|T x-T^{n-1} x\right\| \leq\|x-T x\|+\left\|x-T^{n-1} x\right\| \leq\left(1+k^{n-1}\right)\|x-T x\|,
$$

from this $\gamma_{5}^{H} \geq 1.1986$. 
For $n \geq 6$ we shall also take the following estimations: if $j=0, i=n-2$,

$$
\begin{aligned}
\left\|x-T^{n-2} x\right\| & \leq\left\|x-T^{n-1} x\right\|+\left\|T^{n-2} x-T^{n-1} x\right\| \\
& \leq\left(k^{n-2}+k^{n-1}\right)\|x-T x\|,
\end{aligned}
$$

and if $j=2, i=n-1$,

$$
\left\|T^{2} x-T^{n-1} x\right\| \leq\left\|x-T^{2} x\right\|+\left\|x-T^{n-1}\right\| \leq\left(1+k+k^{n-1}\right)\|x-T x\| .
$$

With this we get $\gamma_{6}^{H} \geq 1.15$.

In the case above we considered $a_{i}=1 / n$ because the calculations are straightforward, but we can choose other convex combinations in order to get better estimations of $\gamma_{n}^{H}$.

Proposition 2.5. Let $H$ be a Hilbert space. Then $\gamma_{3}^{H} \geq 1.5549, \gamma_{4}^{H} \geq$ 1.3267, $\gamma_{5}^{H} \geq 1.2152$, and $\gamma_{6}^{H} \geq 1.1562$.

Proof. Let $F$ be as in Proposition 2.3. We will only take the case in which $F\left(k, a_{j}, a_{j+1}, a_{i}, a_{i+1}\right) \geq 0$ for $1 \leq j<i \leq n-1$.

If $n=3$, we checked by numerical computation that this case gives us the solution with the greatest possible value of $k$. For $n$ larger than 3 , we do not know if this case gives us the best estimate, but it is easier to compute.

For $n=3$, let $z=a_{1} T x+a_{2} T^{2} x+a_{3} x$, where $a_{1}+a_{2}+a_{3}=1$, with $a_{i} \geq 0$. By Proposition 2.3 we have

$$
\begin{aligned}
& \|z-T z\|^{2} \leq F\left(k, a_{3}, a_{1}, a_{1}, a_{2}\right)\|x-T x\|^{2} \\
& \quad+F\left(k, a_{3}, a_{1}, a_{2}, a_{3}\right)\left\|x-T^{2} x\right\|^{2}+F\left(k, a_{1}, a_{2}, a_{2}, a_{3}\right)\left\|T x-T^{2} x\right\|^{2} \\
& \leq F\left(k, a_{3}, a_{1}, a_{1}, a_{2}\right)\|x-T x\|^{2}+k^{4} F\left(k, a_{3}, a_{1}, a_{2}, a_{3}\right)\|x-T x\|^{2} \\
& \quad+k^{2} F\left(k, a_{1}, a_{2}, a_{2}, a_{3}\right)\|x-T x\|^{2}=B\left(k, a_{1}, a_{2}, a_{3}\right)\|x-T x\|^{2} .
\end{aligned}
$$

Using differential calculus techniques, we conclude that the solution of the equation $B\left(k, a_{1}, a_{2}, a_{3}\right)=1$ with the optimal value of $k$ is the following: $k=1.5549978175686, a_{1}=0.22027175125, a_{2}=0.44334559817$ and $a_{3}=$ 0.33638265058 .

Let $n=4$, by Proposition 2.3 and using the estimations of the terms $\left\|T^{j} x-T^{i} x\right\|$ as in the proof of Proposition 2.4, we have to solve

$$
\begin{aligned}
G(k, x, y, z, w)= & F\left(k, a_{4}, a_{1}, a_{1}, a_{2}\right)+(1+k)^{2} F\left(k, a_{4}, a_{1}, a_{2}, a_{3}\right) \\
& +k^{6} F\left(k, a_{4}, a_{1}, a_{3}, a_{4}\right)+k^{2} F\left(k, a_{1}, a_{2}, a_{2}, a_{3}\right) \\
& +k^{2}(1+k)^{2} F\left(k, a_{1}, a_{2}, a_{3}, a_{4}\right) \\
& +k^{4} F\left(k, a_{2}, a_{3}, a_{3}, a_{4}\right)-1=0 .
\end{aligned}
$$


The following optimal solution was found numerically:

$$
\begin{aligned}
k & =1.326774364525014, \\
a_{1} & =0.242229079187726, \\
a_{2} & =0.239942791859123, \\
a_{3} & =0.328255853776722, \\
a_{4} & =0.189572275176429 .
\end{aligned}
$$

Similarly for $n=5$ and using the estimate (2.6), we need to solve

$$
\begin{aligned}
& F\left(k, a_{5}, a_{1}, a_{1}, a_{2}\right)+(1+k)^{2} F\left(k, a_{5}, a_{1}, a_{2}, a_{3}\right) \\
& +k^{6}(1+k)^{2} F\left(k, a_{5}, a_{1}, a_{3}, a_{4}\right)+k^{8} F\left(k, a_{5}, a_{1}, a_{4}, a_{5}\right) \\
& +k^{2} F\left(k, a_{1}, a_{2}, a_{2}, a_{3}\right)+k^{2}(1+k)^{2} F\left(k, a_{1}, a_{2}, a_{3}, a_{4}\right) \\
& +\left(1+k^{4}\right)^{2} F\left(k, a_{1}, a_{2}, a_{4}, a_{5}\right)+k^{4} F\left(k, a_{2}, a_{3}, a_{3}, a_{4}\right) \\
& +k^{4}(1+k)^{2} F\left(k, a_{2}, a_{3}, a_{4}, a_{5}\right)+k^{6} F\left(k, a_{3}, a_{4}, a_{4}, a_{5}\right)-1=0 .
\end{aligned}
$$

We found the optimal solution: $k=1.215236, a_{1}=0.14448498, a_{2}=$ $0.23632485, a_{3}=0.24363867, a_{4}=0.20374357$ and $a_{5}=0.17180793$.

For $n=6$ using (2.6), (2.7) and (2.8), we obtain the equation to solve

$$
\begin{aligned}
& F\left(k, a_{6}, a_{1}, a_{1}, a_{2}\right)+(1+k)^{2} F\left(k, a_{6}, a_{1}, a_{2}, a_{3}\right) \\
& +\left(1+k+k^{2}\right)^{2} F\left(k, a_{6}, a_{1}, a_{3}, a_{4}\right)+\left(k^{5}+k^{4}\right)^{2} F\left(k, a_{6}, a_{1}, a_{4}, a_{5}\right) \\
& +k^{10} F\left(k, a_{6}, a_{1}, a_{5}, a_{6}\right)+k^{2} F\left(k, a_{1}, a_{2}, a_{2}, a_{3}\right) \\
& +k^{2}(1+k)^{2} F\left(k, a_{1}, a_{2}, a_{3}, a_{4}\right)+k^{2}\left(1+k+k^{2}\right)^{2} F\left(k, a_{1}, a_{2}, a_{4}, a_{5}\right) \\
& +\left(1+k^{5}\right)^{2} F\left(k, a_{1}, a_{2}, a_{5}, a_{6}\right)+k^{4} F\left(k, a_{2}, a_{3}, a_{3}, a_{4}\right) \\
& +k^{4}(1+k)^{2} F\left(k, a_{2}, a_{3}, a_{4}, a_{5}\right)+\left(1+k+k^{5}\right)^{2} F\left(k, a_{2}, a_{3}, a_{5}, a_{6}\right) \\
& +k^{6} F\left(k, a_{3}, a_{4}, a_{4}, a_{5}\right)+k^{6}(1+k)^{2} F\left(k, a_{3}, a_{4}, a_{5}, a_{6}\right) \\
& +k^{8} F\left(k, a_{4}, a_{5}, a_{5}, a_{6}\right)-1=0 .
\end{aligned}
$$

We get the following optimal solution: $k=1.1562, a_{1}=0.15958598$, $a_{2}=0.15893532, a_{3}=0.17823298, a_{4}=0.19267723, a_{5}=0.15822986$ and $a_{6}=0.15233863$.

3. $\boldsymbol{T} \in \mathscr{L}(\boldsymbol{k}) \cap \mathscr{U}(\boldsymbol{p})$ with $\boldsymbol{p}<\boldsymbol{k}^{\boldsymbol{n}-\mathbf{1}}$. In Proposition 2.4 we used $T^{j} \in$ $\mathscr{L}\left(k^{j}\right)$ in order to calculate the best estimation of $\gamma_{n}^{H}$. In fact, there are $n$-periodic functions such that for each $j=1, \ldots, n-1, T^{j} \in \mathscr{L}_{0}\left(k^{j}\right)$ that is $T^{j} \notin \mathscr{L}(p)$ for $p<k^{j}$,

Example 3.1. Let $X=c_{0}^{n}(\mathbb{R}), C=\left\{\left(x_{1}, \ldots, x_{n}\right) \in X: x_{i} \geq 0, i=\right.$ $1, \ldots, n\}$ and $k>1$. We define $T: C \rightarrow C$ as follows:

$$
T\left(x_{1}, \ldots, x_{n}\right)=\left(k x_{2}, k x_{3}, \ldots, k x_{n}, \frac{x_{1}}{k^{n-1}}\right) .
$$


We have $T^{n}=I$ and, in fact, for each $j=1, \ldots, n-1, T^{j} \in \mathscr{L}\left(k^{j}\right)$ but $T^{j} \notin \mathscr{L}(p)$ for $p<k^{j}$.

In this case we have $T \in \mathscr{U}_{0}\left(k^{n-1}\right)$ according to the following definition:

Definition 3.2. Let $T: C \rightarrow C$ be a mapping, where $C$ is a nonempty, closed and convex subset of a Banach space. We will say that $T$ is uniformly Lipschitzian if there is $k>0$ such that for every $j$ and $x, y \in C$,

$$
\left\|T^{j} x-T^{j} y\right\| \leq k\|x-y\| .
$$

We will write $T \in \mathscr{U}(k)$. If

$$
k=\min \left\{l:\left\|T^{j} x-T^{j} y\right\| \leq l\|x-y\|, j \in \mathbb{N}, x, y \in C\right\},
$$

we will write $T \in \mathscr{U}_{0}(k)$.

However, there are also cases such that $T$ is $n$-periodic, $T \in \mathscr{L}_{0}(k)$ and $T \in \mathscr{U}_{0}(p)$ with $p<k^{n-1}$. For these functions we could improve the estimations considered in Proposition 2.4.

The extreme case is when $T^{j} \in \mathscr{L}_{0}(k)$ for $1 \leq j \leq n-1$, that is, $T \in$ $\mathscr{U}_{0}(k)$. The next example shows that such functions exist.

Example 3.3. Let $X=c_{0}^{n}(\mathbb{R}), C=\left\{\left(x_{1}, \ldots, x_{n}\right) \in X: x_{i} \geq 0, i=\right.$ $1, \ldots, n\}$ and $k>1$. We define $T: C \rightarrow C$ as follows:

$$
T\left(x_{1}, \ldots, x_{n}\right)=\left(x_{2}, \ldots, x_{n-1}, k x_{n}, \frac{x_{1}}{k}\right) .
$$

We have $T^{n}=I$ and for each $j=1, \ldots, n-1, T^{j} \in \mathscr{L}(k)$.

For this reason, we will introduce the following definition: let $X$ be a Banach space, we define

$$
\tilde{\gamma}_{n}^{X}=\inf \left\{p: \exists(C \subset X, T: C \rightarrow C), T^{n}=I, T \in \mathscr{U}_{0}(p), \operatorname{Fix}(T)=\emptyset\right\},
$$

where $C$ is a nonempty, closed and convex subset of the Banach space $X$. It is clear that $\tilde{\gamma}_{n}^{X} \geq \gamma_{n}^{X}$, since $T \in \mathscr{L}_{0}(k)$ implies $T \in \mathscr{U}_{0}(p)$ with $p \geq k$.

As before we want to estimate $\tilde{\gamma}_{n}^{H}$.

Proposition 3.4. Let $H$ be a Hilbert space. Then $\tilde{\gamma}_{3}^{H} \geq 1.6047, \tilde{\gamma}_{4}^{H} \geq$ 1.3867, $\tilde{\gamma}_{5}^{H} \geq 1.2958$ and $\tilde{\gamma}_{6}^{H} \geq 1.2181$.

Proof. Let $T \in \mathscr{U}(p)$ and $T^{n}=I$. By Proposition 2.3, if we take $z=$ $\sum_{i=1}^{n} a_{i} T^{i} x$, then we have

$$
\|z-T z\|^{2} \leq \sum_{0 \leq j<i \leq n-1}\left[p^{2}\left(a_{j+1} a_{i}+a_{j} a_{i+1}-a_{j} a_{i}\right)-a_{j} a_{i}\right]\left\|T^{j} x-T^{i} x\right\|^{2} .
$$

Let $n \geq 3$ and $d=\|x-T x\|$. We will use the estimates:

(1) $\left\|x-T^{j} x\right\| \leq \min \{((j-1) p+1) d,(n-j) p d\}$,

(2) $\left\|T x-T^{j} x\right\| \leq \min \{(j-1) p d,((n-j) p+1) d\}$,

(3) if $i>1$ and $i+j<n,\left\|T^{i} x-T^{i+j} x\right\| \leq \min \{((n-j-1) p+1) d, p j d\}$. 
As in Proposition 2.5 we only take $F\left(p, a_{j}, a_{j+1}, a_{i}, a_{i+1}\right) \geq 0$ for $0 \leq j<$ $i \leq n-1$. For $n=3$ we know that this is the best possibility but for $n>3$ we do not know if this is the case.

Thus, for $n=3$ we have to solve the equation

$$
F\left(p, a_{3}, a_{1}, a_{1}, a_{2}\right)+p^{2} F\left(p, a_{3}, a_{1}, a_{2}, a_{3}\right)+p^{2} F\left(p, a_{1}, a_{2}, a_{2}, a_{3}\right)=1 .
$$

The solution with the optimal value of $p$ is: $p=1.6047, a_{1}=0.4278208$, $a_{2}=0.34664038$ and $a_{3}=0.22553882$.

For $n=4$ we have to solve

$$
\begin{aligned}
& F\left(p, a_{4}, a_{1}, a_{1}, a_{2}\right)+(1+p)^{2} F\left(p, a_{4}, a_{1}, a_{2}, a_{3}\right) \\
& +p^{2} F\left(p, a_{4}, a_{1}, a_{3}, a_{4}\right)+p^{2} F\left(p, a_{1}, a_{2}, a_{2}, a_{3}\right) \\
& +(1+p)^{2} F\left(p, a_{1}, a_{2}, a_{3}, a_{4}\right)+p^{2} F\left(p, a_{2}, a_{3}, a_{3}, a_{4}\right)-1=0 .
\end{aligned}
$$

The optimal solution is: $p=1.3867, a_{1}=0.30095499, a_{2}=0.23635124$, $a_{3}=0.2667267$ and $a_{4}=0.19596707$.

For $n=5$ we have to solve the equation

$$
\begin{aligned}
& F\left(p, a_{5}, a_{1}, a_{1}, a_{2}\right)+(1+p)^{2} F\left(p, a_{5}, a_{1}, a_{2}, a_{3}\right) \\
& +4 p^{2} F\left(p, a_{5}, a_{1}, a_{3}, a_{4}\right)+p^{2} F\left(p, a_{5}, a_{1}, a_{4}, a_{5}\right) \\
& +p^{2} F\left(p, a_{1}, a_{2}, a_{2}, a_{3}\right)+4 p^{2} F\left(p, a_{1}, a_{2}, a_{3}, a_{4}\right) \\
& +(1+p)^{2} F\left(p, a_{1}, a_{2}, a_{4}, a_{5}\right)+p^{2} F\left(p, a_{2}, a_{3}, a_{3}, a_{4}\right) \\
& +4 p^{2} F\left(p, a_{2}, a_{3}, a_{4}, a_{5}\right)+p^{2} F\left(p, a_{3}, a_{4}, a_{4}, a_{5}\right)-1=0 .
\end{aligned}
$$

The optimal solution is: $p=1.2958, a_{1}=0.20310133, a_{2}=0.19687386$, $a_{3}=0.24013125, a_{4}=0.15037377$ and $a_{5}=0.20951979$.

Finally, for $n=6$ we have the equation

$$
\begin{aligned}
& F\left(p, a_{6}, a_{1}, a_{1}, a_{2}\right)+(1+p)^{2} F\left(p, a_{6}, a_{1}, a_{2}, a_{3}\right) \\
& +(1+2 p)^{2} F\left(p, a_{6}, a_{1}, a_{3}, a_{4}\right)+4 p^{2} F\left(p, a_{6}, a_{1}, a_{4}, a_{5}\right) \\
& +p^{2} F\left(p, a_{6}, a_{1}, a_{5}, a_{6}\right)+p^{2} F\left(p, a_{1}, a_{2}, a_{2}, a_{3}\right) \\
& +4 p^{2} F\left(p, a_{1}, a_{2}, a_{3}, a_{4}\right)+(1+2 p)^{2} F\left(p, a_{1}, a_{2}, a_{4}, a_{5}\right) \\
& +(1+p)^{2} F\left(p, a_{1}, a_{2}, a_{5}, a_{6}\right)+p^{2} F\left(p, a_{2}, a_{3}, a_{3}, a_{4}\right) \\
& +4 p^{2} F\left(p, a_{2}, a_{3}, a_{4}, a_{5}\right)+(1+2 p)^{2} F\left(p, a_{2}, a_{3}, a_{5}, a_{6}\right) \\
& +p^{2} F\left(p, a_{3}, a_{4}, a_{4}, a_{5}\right)+4 p^{2} F\left(p, a_{3}, a_{4}, a_{5}, a_{6}\right) \\
& +p^{2} F\left(p, a_{4}, a_{5}, a_{5}, a_{6}\right)-1=0
\end{aligned}
$$

and the optimal solution is: $p=1.2181, a_{1}=0.1682856, a_{2}=0.14103694$, $a_{3}=0.19292656, a_{4}=0.16166393, a_{5}=0.13527042$ and $a_{6}=0.20081655$. 
Summing up, we present the following two tables:

\begin{tabular}{|l|l|l|l|l|l|}
\hline \multicolumn{5}{|c|}{$T \in \mathscr{L}(k)$} \\
\hline & $\begin{array}{l}\text { M. Koter, } \\
2000\end{array}$ & $\begin{array}{l}\text { Our method } \\
a_{i}=1 / n\end{array}$ & $\begin{array}{l}\text { Our method } \\
\sum a_{i}=1\end{array}$ & & $\begin{array}{l}\text { Górnicki and } \\
\text { Pupka, 2005 }\end{array}$ \\
\hline$\gamma_{3}^{H}$ & 1.3666 & 1.4678 & $\mathbf{1 . 5 5 4 9}$ & $\gamma_{3}^{X}$ & 1.3821 \\
\hline$\gamma_{4}^{H}$ & 1.1962 & 1.2905 & $\mathbf{1 . 3 2 6 7}$ & $\gamma_{4}^{X}$ & 1.2524 \\
\hline$\gamma_{5}^{H}$ & 1.0849 & 1.1986 & $\mathbf{1 . 2 1 5 2}$ & $\gamma_{5}^{X}$ & 1.1777 \\
\hline$\gamma_{6}^{H}$ & 1.0228 & 1.15 & $\mathbf{1 . 1 5 6 2}$ & $\gamma_{6}^{X}$ & 1.1329 \\
\hline
\end{tabular}

\begin{tabular}{|c|l|l|l|l|}
\hline \multicolumn{5}{|c|}{$T \in \mathscr{U}_{0}(p)$} \\
\hline & $\begin{array}{l}\text { M. Koter, } \\
\text { Our method } \\
\sum a_{i}=1\end{array}$ & & $\begin{array}{l}\text { Górnicki and } \\
\text { Pupka, 2005 }\end{array}$ \\
\hline$\tilde{\gamma}_{3}^{H}$ & 1.5447 & $\mathbf{1 . 6 0 4 7}$ & $\tilde{\gamma}_{3}^{X}$ & 1.4558 \\
\hline$\tilde{\gamma}_{4}^{H}$ & 1.2418 & $\mathbf{1 . 3 8 6 7}$ & $\tilde{\gamma}_{4}^{X}$ & 1.2917 \\
\hline$\tilde{\gamma}_{5}^{H}$ & 1.1429 & $\mathbf{1 . 2 9 5 8}$ & $\tilde{\gamma}_{5}^{X}$ & 1.2001 \\
\hline$\tilde{\gamma}_{6}^{H}$ & 1.0277 & $\mathbf{1 . 2 1 8 1}$ & $\tilde{\gamma}_{6}^{X}$ & 1.1482 \\
\hline
\end{tabular}

\section{REFERENCES}

[1] Goebel, K., Kirk, W. A., Topics in Metric Fixed Point Theory, Cambridge University Press, Cambridge, 1990.

[2] Goebel, K., Złotkiewicz, E., Some fixed point theorems in Banach spaces, Colloq. Math. 23 (1971), 103-106.

[3] Górnicki, J., Pupka, K., Fixed points of rotative mappings in Banach spaces, J. Nonlinear Convex Anal. 6(2) (2005), 217-233.

[4] Kaczor, W., Koter-Mórgowska M., Rotative mappings and mappings with constant displacement, Handbook of Metric Fixed Point Theory, Kluwer Academic Publisher, Dordrecht, 2001, 323-337.

[5] Koter-Mórgowska, M., Rotative mappings in Hilbert space, J. Nonlinear Convex Anal. 1(3) (2000), 295-304.

H. Fetter Nathansky Centro de Investigacion en Matematicas (CIMAT) Apdo. Postal 402 36000, Guanajuato Gto. Mexico e-mail: fetter@cimat.mx

Received December 18, 2009

\author{
V. Pérez García \\ Centro de Investigacion \\ en Matematicas (CIMAT) \\ Apdo. Postal 402 \\ 36000, Guanajuato \\ Gto. Mexico \\ e-mail: vicpega@cimat.mx
}

